\title{
How OpenStreetMap Is Changing the World
}

\author{
Steve Coast \\ Microsoft \\ One Microsoft way, Redmond, WA 98052, USA \\ steve@asklater.com
}

\begin{abstract}
This talk reviews these and many more aspects of OpenStreetMap and gives an in depth feel of the past, present and future of the project.
\end{abstract}

OpenStreetMap is a map of the world built by volunteers. Using a peer production model similar to wikipedia approximately 300,000 people collaboratively map features such as roads, parks and buildings and submit them to be shared. That map data is made available under an open license for anybody to use as they see fit, with two conditions. First that if you use the maps you must say where they came from and second that you release any changes to the maps under the same creative commons license, CCBYSA.

The project was founded in 2004 as a practical solution to the lack of freely available map data. Map data is traditionally collected and distributed by private enterprises and governments which tend to charge high prices and restrict the usage rights of the data carefully, much as encyclopedia britannica does in contrast to the open wikipedia model. OpenStreetMap is very similar in that regard.

Data is collected in an ad hoc and undirected manner by volunteers worldwide. These volunteers use an open tagging system to classify data as they see fit, instead of a hierarchical top-down ontological system. This leads to man benefits such as allowing mallets to map what they want, when they want. This openness is key to putting as few barriers as possible between mallets and the map.

OpenStreetMap has been key in places such as Haiti post-earthquake where it was used as the defacto map base between government agencies, NGOs and rescue teams in place of clumsy closed and overlapping maps. This has been claimed to save lives. 